\title{
An Autophagy-Related Gene Signature Predicts Prognosis of Early Stage Colorectal Cancer
}

\section{Yi-feng Zou}

Sun Yat-sen University Sixth Affiliated Hospital

\section{Qiu-ning Wu}

Sun Yat-sen University Sixth Affiliated Hospital

Si Qin

Sun Yat-sen University Sixth Affiliated Hospital

Xi Xi Chen

Sun Yat-sen University Sixth Affiliated Hospital

Jia-wei Cai

Sun Yat-sen University Sixth Affiliated Hospital

Ying-xin Tan

Sun Yat-sen University Sixth Affiliated Hospital

Jing-rong Weng

Sun Yat-sen University Sixth Affiliated Hospital

Yu-ming Rong

Sun Yat-sen University Cancer Center

Xutao Lin ( $\square$ linxutao@mail2.sysu.edu.cn )

Sun Yat-sen University Sixth Affiliated Hospital https://orcid.org/0000-0002-7110-3459

\section{Research article}

Keywords: colorectal cancer, prognosis, autophagy-related gene, early stage, disease-free survival

Posted Date: August 26th, 2020

DOI: https://doi.org/10.21203/rs.3.rs-59367/v1

License: () (1) This work is licensed under a Creative Commons Attribution 4.0 International License. Read Full License 


\section{Abstract}

\section{Background and aims}

A certain number of early-stage colorectal cancer (CRC) patients suffer tumor recurrence after initial curative resection. In this context, an effective prognostic biomarker model is constantly in need. Autophagy exhibits a dual role in tumorigenesis. Our study aims to develop an autophagy-related gene (ATG) signature based on high-throughput data analysis for disease-free survival (DFS) prognosis of patients with stage I/II CRC.

\section{Methods}

Gene expression profiles and clinical information of CRC patients extracted from four public datasets were divided into training cohort (GSE39582, $n=566$ ), validation cohort (TCGA CRC, $n=624$ ), and meta-validation cohort (GSE37892 and GSE14333, $n=420$ ). Autophagy genes significantly associated with prognosis were identified.

\section{Results}

Among 655 autophagy-related genes, a 10 gene ATG signature, which was significantly associated with DFS in the training cohort (HR, 2.76[1.56-4.82]; $\left.P=2.06 \times 10^{-4}\right)$, was constructed. The ATG signature, stratifying patients into high and low autophagy risk groups, was validated in the validation $\left(H R, 2.29[1.15-4.55] ; P=1.5 \times 10^{-2}\right)$ and the meta-validation cohorts $\left(H R, 2.5[1.03-6.06] ; P=3.63 \times 10^{-2}\right)$, and proved to be prognostic in a multivariate analysis. Functional analysis revealed enrichment of several immune/ inflammatory processes in the high autophagy risk group, where significantly higher infiltration of T regulatory cells (Tregs) was observed.

\section{Conclusions}

Our study established a prognostic ATG signature that effectively predicted DFS for early-stage CRC patients. Meanwhile, the study also revealed the possible correlation between autophagy and immune/ inflammatory processes and tumorigenesis.

\section{Background}

CRC is currently the second leading cause of cancer deaths worldwide, ranking third in morbidity[1]. Depending upon the tumor stage at diagnosis, relative 5 -year survival rates for patients with CRC range from $65 \%$ for all stages to $91 \%, 82 \%$, and $12 \%$ for patients diagnosed with stage I, II, and IV, respectively[2]. Although increasing awareness of cancer screening and advances in technology have improved early detection[3] and treatment without chemotherapy, around one-third of patients with stages I-III CRC still encounter tumor relapse after curative treatment[4]. Accordingly, there is a growing need for novel molecular markers for prognosis patterns, which might provide valuable information for supplementary adjuvant chemotherapy or other targeted therapy. Recently, for example, the predictive potency of KRAS and BRAF mutations, microsatellite instability (MSI) status, and CpG island methylator phenotype (CIMP) status in CRC had been studied extensively[3, 5].

Autophagy plays a dual role in tumorigenesis[6]. It inhibits early tumor initiation by the clearance of damaged mitochondria, peroxisome, and other cytotoxic substance, and also caters to the high metabolic demands from accelerated proliferating tumors by degrading intracellular organelle and macromolecule substances[7, 8]. To further investigate how autophagy affects the prognosis of early-stage CRC patients, we identified an ATG signature from CRC-specific transcriptomes based on high-throughput data analysis. The ATG signature, which stratified the stage I/II CRC patients into distinct risk groups, together with functional and pathway analysis, might provide insights into the mechanism of $\mathrm{CRC}$ recurrence and targeted treatment.

\section{Methods}

\subsection{Public datasets}

Gene expression data of CRC tissue samples and corresponding clinical information obtained from Gene Expression Omnibus (GEO http://www.ncbi.nlm.nih.gov/geo/) and Firehose Broad GDAC portal (http://gdac.broadinstitute.org/) were retrospectively analyzed. A total of 1610 patient samples from four independent public cohorts were included. The GSE39582 dataset $(n=566)$ was intended for training, among which 309 patients without chemotherapy adjuvant but with survival information served as the discovery cohort. The Cancer Genome Atlas (TCGA) CRC dataset ( $n=624$ ) was used for independent validation, while the GSE37892 and GSE14333 dataset $(n=420)$ were combined for meta-validation. Transcripts per million (TPM) of level 3 RNA-Seq data in the log2 scale were applied to 
calibrate the gene expression levels in the TCGA cohort. The 'combat' algorithm of the R package 'sva' and the z-scores were used to correct the batch effects, to standardize microarray data across multiple experiments, and compare them independent of the original hybridization intensities.

\subsection{Construction of a prognostic autophagy-related gene (ATG) signature}

To construct a prognostic ATG signature, we focused on 655 ATGs from 8 gene sets identified via MSigDB (version 6.2)[9-11] with the keyword "autophagy". Only 617 genes measured on all platforms involved in this study with high variation (determined by median absolute deviation (MAD) >0.5) were selected. After 1000 times of random Cox univariate regressions, genes with repeated significance, which indicated a strong relationship between ATGs and patients' DFS, were selected as candidates for the signature. A Cox proportional hazards regression model on CRC samples together with the least absolute shrinkage and selection operator (LASSO) were applied to minimize the risk of over-fitting as well as to generate a risk model.

Patients were divided into low and high autophagy risk groups in accordance with the optimal ATG signature cutoff, which was defined by the time-dependent receiver operating characteristic (ROC) curve analysis at 5 years of disease-free survival (DFS) in the training cohort. The ATG signature by the shortest distance between the ROC curve and the point at $100 \%$ true positive rate and a $0 \%$ false-positive rate was deemed as the cutoff value.

\subsection{Validation of ATG signature}

C-index was employed in the training, independent validation, and meta-validation cohorts respectively to assess the predictive capability of the model. For further validation, the prognostic value of the ATG signature was evaluated in CRC patients with early stages (stage I\&II) and all stages in different cohorts through survival analysis. Univariate and multivariate analyses of the ATG signature and available clinical parameters was performed to assess whether the ATG signature is an independent risk factor.

\subsection{Functional analysis}

Enrichment of potential pathways of the ATG signature by gene ontology (GO) analysis was performed on gProfiler (https://biit.cs.ut.ee/gprofiler/), and gene set enrichment analysis (GSEA)[12] was conducted using the Bioconductor package'fgsea'. Gene sets of cancer hallmarks from MSigDB with statistical significance (FDR-adjusted $\mathrm{P}<.05$ ) were selected[13]. CIBERSORT[14] was used to dissect immune cell infiltration in different risk groups.

\subsection{Statistical analysis}

All statistical analyses were performed in R software (version 3.5.1). Categorical variables were reported as count. Continuous data were reported as mean with standard deviation (SD) and compared with the student's t-test. The LASSO regression was plotted using the 'glmnet' R package (version: 2.0-16). Time-dependent ROC curve analysis was done by the R package 'survivalROC' (version: 1.0.3). Survival analysis was conducted using the Kaplan-Meier method and compared with the log-rank test. Univariate and multivariate analysis of ATG signature and clinical parameters were performed using the log-rank test. The statistical significance level was set at $\mathrm{a}=0.05$, two-sided.

\section{Results}

\subsection{ATG signature establishment}

After filtration with $M A D>0.5,617$ genes measured on all platforms were selected for this study. By 1000 times of random Cox univariate regressions, 58 ATGs were identified to be strongly relevant to DFS and considered as candidates for the signature (Figure 1). A LASSO Cox regression in stage I and II patients in the training cohort (Table 1) revealed 10 ATGs for the risk model (Figure 2), with coefficient of each ATG listed in Table 2. The risk model was formulated as follow: Autophagy-related risk score $=0.040346631 \times \exp (\mathrm{mRNA}$ expression level of CD163L1) + 0.040346631x exp (mRNA expression level of FAM13B) + 0.160103165x exp (mRNA expression level of HDAC6) + $0.05063732 \times \exp (\mathrm{mRNA}$ expression level of HPR) - 0.012205947× exp (mRNA expression level of NR2C2) - 0.027104325× exp (mRNA 
expression level of RAB12) + 0.055095935× exp (mRNA expression level of SIRT2) - 0.084226324× exp (mRNA expression level of TBC1D14) - 0.012613054× exp (mRNA expression level of TLK2) - 0.001301898x exp (mRNA expression level of TBC1D12).

Based on the time-dependent ROC curve analysis at 5 year-DFS in the training cohort, the optimal cutoff of ATG signature that divided the patients into high and low autophagy risk groups was defined as -0.009 (Figure 3). The risk scores of all patients are shown in Supplementary Table 1. Survival analysis showed the DFS rate was higher in the low autophagy risk group compared to the high autophagy risk group for patients with early stages (stage I and II) in the training cohort (GSE39582) (Figure 4A, 4B, 4C, HR, 2.76[1.564.82]; $P=2.06 \times 10^{-4}$ ). So was indicated for patients in all stages in the GSE39582 dataset (Figure $5 \mathrm{~A}, 5 \mathrm{~B}, 5 \mathrm{C}, \mathrm{HR}, 1.7[1.25-2.31]$; $P=5.21 \times 10^{-}$ $\left.{ }^{4}\right)$. 
Table 1

Characteristics of training (GSE39582), validation (TCGA) and meta-validation cohorts

\begin{tabular}{|c|c|c|c|}
\hline & $\begin{array}{l}\text { GSE39582 } \\
\text { (training) }\end{array}$ & $\begin{array}{l}\text { TCGA } \\
\text { (validation) }\end{array}$ & Meta-validation \\
\hline Number of patients & 566 & 624 & 420 \\
\hline Patients with survival data & 557 & 509 & 356 \\
\hline Mean age, years & $66.85 \pm 13.29$ & $66.27 \pm 12.76$ & $66.66 \pm 12.60$ \\
\hline \multicolumn{4}{|l|}{ Gender, $n$} \\
\hline Male & 310 & 332 & 233 \\
\hline Female & 256 & 292 & 187 \\
\hline \multicolumn{4}{|l|}{ TNM stage, $n$} \\
\hline Stage I & 33 & 105 & 44 \\
\hline Stage II & 264 & 230 & 167 \\
\hline Stage III & 205 & 180 & 148 \\
\hline Stage IV & 60 & 88 & 61 \\
\hline NA & 4 & 21 & - \\
\hline \multicolumn{4}{|l|}{ CMS system, $\mathrm{n}$} \\
\hline CMS1 & 91 & 68 & 74 \\
\hline CMS2 & 232 & 207 & 168 \\
\hline CMS3 & 69 & 64 & 69 \\
\hline CMS4 & 127 & 117 & 97 \\
\hline NA & 47 & 168 & 12 \\
\hline \multicolumn{4}{|l|}{ Tumor location } \\
\hline Left & 342 & 354 & 233 \\
\hline Right & 224 & 270 & 185 \\
\hline NA & - & - & 2 \\
\hline \multicolumn{4}{|l|}{ RFS event, n } \\
\hline Yes & 177 & 100 & 87 \\
\hline No & 380 & 416 & 269 \\
\hline NA & 9 & 108 & 64 \\
\hline \multicolumn{4}{|l|}{ OS event, n } \\
\hline Yes & 191 & 67 & NA \\
\hline No & 371 & 557 & NA \\
\hline NA & 4 & - & 420 \\
\hline \multicolumn{4}{|l|}{ DFS event, $n$} \\
\hline Yes & 248 & 146 & 87 \\
\hline No & 314 & 386 & 269 \\
\hline NA & 4 & 92 & 64 \\
\hline MMR status, $\mathrm{n}$ & & & \\
\hline
\end{tabular}




\begin{tabular}{|c|c|c|c|}
\hline MSI & 75 & 189 & - \\
\hline MSS & 444 & 431 & - \\
\hline NA & 47 & 4 & 420 \\
\hline \multicolumn{4}{|c|}{ CIMP status, n } \\
\hline Positive & 91 & - & - \\
\hline Negative & 405 & - & - \\
\hline NA & 70 & 624 & 420 \\
\hline \multicolumn{4}{|c|}{ CIN status, $\mathrm{n}$} \\
\hline Positive & 353 & - & - \\
\hline Negative & 110 & - & - \\
\hline NA & 103 & 624 & 420 \\
\hline \multicolumn{4}{|c|}{ TP53 status, $\mathrm{n}$} \\
\hline Wild type & 161 & - & - \\
\hline Mutation & 190 & - & - \\
\hline NA & 215 & 624 & 420 \\
\hline \multicolumn{4}{|c|}{ KRAS status, $\mathrm{n}$} \\
\hline Wild type & 328 & 34 & - \\
\hline Mutation & 217 & 30 & - \\
\hline NA & 21 & 560 & 420 \\
\hline \multicolumn{4}{|c|}{ BRAF status, $\mathrm{n}$} \\
\hline Wild type & 461 & 32 & - \\
\hline Mutation & 51 & 3 & - \\
\hline NA & 54 & 589 & 420 \\
\hline \multicolumn{4}{|c|}{ Chemotherapy adjuvant, $\mathrm{n}$} \\
\hline Yes & 233 & 231 & 118 \\
\hline No & 316 & 393 & 171 \\
\hline NA & 17 & - & 131 \\
\hline
\end{tabular}


Table 2

Model information.

\begin{tabular}{|lllll|}
\hline Gene & Name & $\begin{array}{l}\text { Frequency } \\
\text { in resampling }\end{array}$ & $\begin{array}{l}\text { Average } \\
\text { P-value }\end{array}$ & Coefficient \\
\hline CD163L1 & CD163 molecule like 1 & 674 & 0.050594905 & 0.040346631 \\
\hline FAM13B & family with sequence similarity 13 member B & 625 & 0.050594905 & 0.040346631 \\
\hline HDAC6 & histone deacetylase 6 & 719 & 0.042438619 & 0.160103165 \\
\hline HPR & haptoglobin-related protein & 769 & 0.051952291 & 0.05063732 \\
\hline NR2C2 & nuclear receptor subfamily 2 group C member 2 & 898 & 0.019300065 & -0.012205947 \\
\hline RAB12 & RAB12, member RAS oncogene family & 866 & 0.025407812 & -0.027104325 \\
\hline SIRT2 & sirtuin 2 & 877 & 0.023493248 & 0.055095935 \\
\hline TBC1D14 & TBC1 domain family member 14 & 998 & 0.002105817 & -0.084226324 \\
\hline TLK2 & tousled like kinase 2 & 743 & 0.040418795 & -0.012613054 \\
\hline TBC1D12 & TBC1 domain family member 12 & 999 & 0.002072649 & -0.001301898 \\
\hline
\end{tabular}

\subsection{Validation of the ATG signature}

To assess the predictive capability of the risk model, C-index was first applied to various cohorts which turned out to be $0.74(95 \% \mathrm{Cl}, 0.63$ $0.85)$ in the GSE39582 cohort, $0.70(95 \% \mathrm{Cl}, 0.54-0.85)$ in the TCGA cohort and $0.70(95 \% \mathrm{Cl}, 0.51-0.89)$ in the meta-validation cohort (Table 3), higher than those of Oncotype DX colon. To further verify whether the ATG signature predicted well in various populations, we employed the same formula to the independent validation cohort (TCGA) and the meta-validation cohort (GSE37892 and GSE14333). Patients were significantly stratified into different risk groups by the ATG signature considering DFS. For early stages, CRC patients in high autophagy risk group displayed poorer DFS in both the independent validation cohort (Figure 4D, 4E, 4F, HR, 2.29[1.15-4.55]; $P=1.5 \times 10^{-2}$ ) and the meta-validation cohort (Figure 4G, 4H, 4I, HR, 2.5[1.03-6.06]; $P=3.63 \times 10^{-2}$ ). So was indicated for patients with all stages in both the independent validation (Figure 5D, 5E, 5F, HR, 1.79[1.16-2.7]; $P=5.3 \times 10^{-3}$ ) and the meta-validation cohorts (Figure 5G, 5H, 5I, HR, 1.64[1.04-2.52]; $P=3.16 \times 10^{-2}$ ). Besides, univariate and multivariate analysis further proved ATG as an independent prognostic factor after adjusting for clinical parameters such as sex and tumor stage (Table 4).

Table 3

C-index for autophagy risk compared with Oncotype DX colon in three cohorts

\begin{tabular}{|lllll|}
\hline Cohorts & \multicolumn{2}{c}{ Autophagy Risk } & \multicolumn{2}{c|}{ Oncotype DX colon } \\
\cline { 2 - 5 } & C-index & $95 \% \mathrm{Cl}$ & C-index & $95 \% \mathrm{Cl}$ \\
\hline GSE39582 (training) & 0.74 & $0.63-0.85$ & 0.65 & $0.53-0.77$ \\
\hline TCGA (validation) & 0.70 & $0.54-0.85$ & 0.61 & $0.44-0.77$ \\
\hline Meta-validation & 0.70 & $0.51-0.89$ & 0.62 & $0.43-0.82$ \\
\hline TCGA, the Cancer Genome Atlas. & & & \\
\hline
\end{tabular}


Table 4

Univariate and multivariate analysis of ATG signature, clinical and pathologic factors in validation cohorts

\begin{tabular}{|c|c|c|c|c|c|c|c|c|c|c|c|c|}
\hline \multirow[t]{3}{*}{ Characteristic } & \multicolumn{4}{|c|}{ GSE39582 (training) } & \multicolumn{4}{|c|}{ TCGA(validation) } & \multicolumn{4}{|c|}{ Meta-validation } \\
\hline & \multicolumn{2}{|c|}{ Univariate } & \multicolumn{2}{|c|}{ Multivariate } & \multicolumn{2}{|c|}{ Univariate } & \multicolumn{2}{|c|}{ Multivariate } & \multicolumn{2}{|c|}{ Univariate } & \multicolumn{2}{|c|}{ Multivariate } \\
\hline & $\begin{array}{l}\text { HR } \\
(95 \% \\
\mathrm{Cl})\end{array}$ & $\mathrm{P}$ & $\begin{array}{l}\text { HR } \\
(95 \% \\
\mathrm{Cl})\end{array}$ & $\mathrm{P}$ & $\begin{array}{l}\text { HR } \\
(95 \% \\
\mathrm{Cl})\end{array}$ & $\mathrm{P}$ & $\begin{array}{l}\text { HR } \\
(95 \% \\
\mathrm{Cl})\end{array}$ & $\mathrm{P}$ & $\begin{array}{l}\text { HR } \\
(95 \% \\
\mathrm{Cl})\end{array}$ & $\mathrm{P}$ & $\begin{array}{l}\mathrm{HR} \\
(95 \% \mathrm{Cl})\end{array}$ & $\mathrm{P}$ \\
\hline $\begin{array}{l}\text { ATG } \\
\text { signature }\end{array}$ & $\begin{array}{l}2.76 \\
(1.58- \\
4.82)\end{array}$ & 0.00021 & $\begin{array}{l}2.66 \\
(1.52- \\
4.65)\end{array}$ & 0.0006 & $\begin{array}{l}2.27 \\
(1.14 \\
- \\
4.51)\end{array}$ & 0.016 & $\begin{array}{l}2.27 \\
(1.14 \\
- \\
4.51)\end{array}$ & 0.016 & $\begin{array}{l}2.50 \\
(1.03- \\
6.08)\end{array}$ & 0.036 & $\begin{array}{l}2.50 \\
(1.03- \\
6.08)\end{array}$ & 0.036 \\
\hline Sex & $\begin{array}{l}1.51 \\
(0.87- \\
2.61)\end{array}$ & 0.14 & & & $\begin{array}{l}1.29 \\
(0.65 \\
- \\
2.53)\end{array}$ & 0.46 & & & $\begin{array}{l}0.86 \\
(0.38 \text { - } \\
1.96)\end{array}$ & 0.73 & & \\
\hline Age & $\begin{array}{l}1.01 \\
(0.99- \\
1.03)\end{array}$ & 0.4 & & & $\begin{array}{l}1.01 \\
(0.98 \\
- \\
1.04)\end{array}$ & 0.46 & & & $\begin{array}{l}0.97 \\
(0.94- \\
1.00)\end{array}$ & 0.08 & & \\
\hline $\begin{array}{l}\text { Tumor } \\
\text { location }\end{array}$ & $\begin{array}{l}1.09 \\
(0.63- \\
1.88)\end{array}$ & 0.75 & & & $\begin{array}{l}0.94 \\
(0.48 \\
- \\
1.83)\end{array}$ & 0.85 & & & $\begin{array}{l}1.28 \\
(0.55- \\
2.96)\end{array}$ & 0.56 & & \\
\hline TNM stage & $\begin{array}{l}7.61 \\
(1.07- \\
53.86)\end{array}$ & 0.016 & $\begin{array}{l}7.19 \\
(1.01- \\
51.32)\end{array}$ & 0.049 & $\begin{array}{l}1.74 \\
(0.72 \\
- \\
4.20)\end{array}$ & 0.22 & & & $\begin{array}{l}2.67 \\
(0.63- \\
11.38)\end{array}$ & 0.17 & & \\
\hline MMR status & $\begin{array}{l}1.60 \\
(0.68- \\
3.75)\end{array}$ & 0.28 & & & $\begin{array}{l}0.78 \\
(0.40 \\
- \\
1.54)\end{array}$ & 0.47 & & & & & & \\
\hline CIMP positive & $\begin{array}{l}0.99 \\
(0.46- \\
2.11)\end{array}$ & 0.97 & & & & & & & & & & \\
\hline CIN positive & $\begin{array}{l}1.60 \\
(0.71- \\
3.62)\end{array}$ & 0.26 & & & & & & & & & & \\
\hline $\begin{array}{l}\text { TP53 } \\
\text { mutation }\end{array}$ & $\begin{array}{l}1.40 \\
(0.77- \\
2.54)\end{array}$ & 0.26 & & & & & & & & & & \\
\hline $\begin{array}{l}\text { KRAS } \\
\text { mutation }\end{array}$ & $\begin{array}{l}1.26 \\
(0.74- \\
2.13)\end{array}$ & 0.4 & & & $\begin{array}{l}0.82 \\
(0.17 \\
- \\
4.07)\end{array}$ & 0.81 & & & & & & \\
\hline
\end{tabular}

ATG, autophagy-related gene. MMR, mismatch repair. CIMP, CpG island methylator phenotype. CIN, chromosomal instability. HR, hazard ratio. $\mathrm{Cl}$, confident interval.

\subsection{Functional analysis of the ATG signature}

GO analysis and GSEA were carried out to explore the biological function and signaling pathways of genes from the ATG signature. GO analysis revealed that the genes within the ATG signature were mostly involved in the regulation of autophagy and catabolic processes (Figure 6A and Supplementary Table 2). GSEA was performed between different risk groups to further investigate the pathways that were significantly affected. We found a significant enrichment in multiple immune/ inflammatory pathways in the high autophagy risk group, including the IL6/JAK/STAT3 signaling pathway, the IL2/STAT5 pathway, the IFN-apathway, the IFN-ץ pathway, the inflammatory response 
pathway (Figure 6B, P value<0.005). Some cell cycle/ metabolism associated pathways, including the G2-M, oxidative phosphorylation, E2F, MYC were also significantly enriched in the high autophagy risk group, in addition to a few classic pathways like mTORC1 and epithelial-mesenchymal transmission (EMT; P value<0.005).

As there was a significant enrichment in the immune/ inflammatory pathway through GSEA analysis, we conducted immune infiltration analysis. ESTIMATE algorithm displayed significant differences in the immune score $(P=0.02)$ and ESTIMATE score $(P=0.027)$ between high and low autophagy risk groups in the TCGA CRC cohort (Figure 7A). Infiltration of plasma cells and Tregs were enriched significantly in the high autophagy risk group compared with the low autophagy risk group in the GSE39582 and TCGA cohort (Figure 7C). By contrast, M1 macrophage infiltration turned out to be significantly lower in the high autophagy risk group (Figure 7C).

\subsection{Adjuvant chemotherapy effects on different autophagy risk groups}

Survival analysis conducted for different autophagy risk groups with and without adjuvant chemotherapy respectively, showed that for early stage CRC patients without adjuvant chemotherapy in the high autophagy risk group displayed poorer DFS in the GSE39582 cohort (Figure 8A, 5[2.38-10.5]; $\mathrm{P}=2.42 \times 10^{-6}$ ). However, DFS showed no significant difference between high and low autophagy risk groups for early CRC patients with adjuvant chemotherapy (Figure 8B, $P=4.46 \times 10^{-1}$ ). Similar results were observed in the TCGA cohort (Figure 8C, 2.05[0.9-4.67]; $P=8.22 \times 10^{-2}$ and Figure $\left.8 D, P=5.84 \times 10^{-1}\right)$.

\section{Discussion}

Thanks to the improved awareness of cancer screening, CRC is now detected at an early stage, resulting in a better rate of survival. Surgery without chemotherapy, which was deemed as the curative treatment, was carried out on the majority of patients with stage I/II colon cancer and in some cases of stage I/II rectal cancer[2]. Indeed, it enabled prevention from unnecessary side effects of chemotherapy. Nevertheless, more than $20 \%$ of patients with stage I/II CRC who underwent surgical resection still suffered recurrence[13]. Quite a few multigene prognostic signatures have been developed for CRC, but none of them graduated to widespread application due to the uncertainty of prognostic accuracy. Accordingly, an effective prognostic model composed of multiple biomarkers to distinguish earlystage patients with a high risk of recurrence is crucial and necessary for elective adjuvant chemotherapy or other targeted treatments.

Emerging studies have revealed that autophagy functions diversely in the development, maintenance, and progression of tumors. While autophagy may prevent cellular cancerous transformation in normal tissue, it acts as a survival mechanism in established tumors, especially under stress conditions and in response to chemotherapy[14]. Several autophagy inhibitors and activators have been brought up as improved chemotherapeutic options for cancer treatment[15], but without sufficient clinically significant results, especially in CRC. Accordingly, further investigation on the biological mechanism of autophagy in the tumor microenvironment deserves attention, and more targets associated with autophagy awaits to be found.

In our study, we developed a prognostic model comprised of 10 ATGs for stage I/II CRC. This ATG signature, which classified patients into high and low autophagy risk groups, demonstrated a significant difference in 5-year DFS. The C-index of the ATG signature exhibited a good clinical predicting fitness superior to Oncotype DX colon. Validation results suggested that the ATG signature could successfully predict DFS for stage I/II CRC patients after treatment. This novel model enabled us to identify CRC patients with high autophagy risk stood for increased risk of tumor recurrence. Surprisingly, despite the original intention for DFS prediction of early-stage CRC patients, the ATG signature also showed a significant effect in the prediction for all stages. Therefore, this model could be applied to predict tumor recurrence in all CRC patients independent of tumor stage. Upon the prognostic prediction, clinicians could thus make an informed decision regarding supplementary treatment regimens. For example, adjuvant chemotherapy might improve the outcome of CRC patients with high autophagy risk, for there was no significant difference in DFS between the two autophagy risk groups after adjuvant chemotherapy in this study. It might reduce the impact of autophagy on tumor relapse through massive, indiscriminate cell killing, and immunosuppression.

As we looked over the genes within the ATG signature, some of them have been found correlated to CRC, but mostly bear contextdependent biological functions in cancers, similar to autophagy. For example, the cytosolic histone deacetylase 6 (HDAC6) served as a tumor suppressor in hepatocellular carcinogenesis[16], while another study revealed HDAC6 inhibitor significantly suppressed the proliferation and viability, and induced apoptosis in CRC cells, where autophagy activation was observed[17]. Elevated Sirtuin 2 (SIRT2) was found to be associated with poor prognosis in CRC patients via its participation in tumor angiogenesis[18]. Meanwhile, in a separate study SIRT2 was found to be downregulated in colon cancer biopsies compared to adjacent noncancerous tissues, and overexpression of SIRT2 inhibited the proliferation and metastatic progression of SW480 cells[19]. In terms of autophagy-related functions, a previous

Page 9/18 
investigation reported that in response to oxidative stress or serum starvation, SIRT2 dissociated as acetylated FOX01, which later bound to autophagy protein 7 (ATG7) and induced autophagy in tumors[20]. As these inconsistencies make it difficult to clarify the role of autophagy in CRC, we further investigated the biological functions of the ATG signature, expecting to find some clues in the autophagyrelated functions in tumors.

GSEA revealed that the ATG signature included genes that were robustly involved in multiple immune/ inflammatory pathways including IL6/JAK/STAT3, IL2/STAT5, IFN-a, IFN-y, TNF-a/NF-KB, and the inflammatory response presented a particular relation to CRC proliferation or prognosis as previous studies revealed[21-25]. As our findings suggested that a high autophagy risk score correlated with the downregulation of these immune/ inflammatory pathways, we speculated that autophagy might play a role in CRC tumorigenesis and tumor proliferation via an anti-immune/ anti-inflammatory response. Moreover, increased infiltration of Tregs and decreased infiltration of M1 macrophages observed in the high autophagy risk group during immune infiltration analysis seemingly catered to the anti-immune/ antiinflammatory response. No significant difference in cytotoxic T lymphocyte was found in the immune infiltration analysis. Tregs are known to suppress both antibody-mediated and cell-mediated immune responses[26]. The pro-inflammatory M1 macrophages, a phenotype of tumor-associated macrophages (TAMs), correlated to a better prognosis in CRC patients for its tumor-suppressing function[27, 28]. By triggering an anti-immune or anti-inflammatory response, autophagy might promote polarization or re-polarization towards the M2 phenotype spontaneously and thus lead to the decrease of M1 infiltration observed. Previous studies have described the link between autophagy and macrophage polarization in tumor microenvironment. For example, mTOR which functions as a conserved kinase protein in the regulation of autophagy also participates in the polarization of monocytes into TAMs[29]. More new strategies targeting TAM polarization as well as autophagy await exploration and further studies are needed to clarify the above speculations.

However, our prognostic ATG signature relies on the gene expression profiles from microarray platforms, which make it too expensive and time-consuming to popularize in clinical application. In addition to the dataset limitations from retrospective studies, further prospective clinical tests are recommended to validate our results. Despite the limitations, our research proposes a novel perspective to predicting the prognosis of early CRC patients and offers valuable insights into the relationship between autophagy, immune/ inflammatory response, and tumorigenesis.

\section{Conclusion}

In conclusion, our study established a prognostic ATG signature that can effectively predict DFS for early-stage CRC patients. Meanwhile, we also revealed the possible correlation between ATG signature and immune/ inflammatory pathways.

\section{Abbreviations}

CRC, colorectal cancer; ATG, autophagy-related gene; DFS, disease-free survival; TCGA, The Cancer Genome Atlas; TAM, tumor-associated macrophage; Tregs, T regulatory cells; LASSO, the least absolute shrinkage and selection operator; ROC, receiver operating characteristic; $\mathrm{GO}$, gene ontology.

\section{Declarations}

\subsection{Ethics approval and consent to participate}

Not applicable.

\subsection{Consent for publication}

Not applicable.

\subsection{Availability of data and materials}

The datasets analyzed for this study can be found in the Gene Expression Omnibus (GEO http://www.ncbi.nlm.nih.gov/geo/) and Firehose Broad GDAC portal (http://gdac.broadinstitute.org/).

\subsection{Competing interests}




\subsection{Funding}

This study was supported by grants from National Key Clinical Discipline, the Fundamental Research Funds for the young teacher training program of Sun Yat-sen University (grant number 18ykpy02).

\subsection{Authors' contributions}

$Y Z, Y R$ and $X L$ contributed the conception and design of the study; $Y Z, S Q$ and $X L$ collected the datasets; $Y Z$, QW, SQ and XC performed the statistical analysis; $Y Z, Q W, S Q, X C, J C, Y T, J W, Y R$ and $X L$ participated in the data interpretation; $Q W$ wrote the first draft of the manuscript; $Y Z, J C, Y R$, XL wrote sections of the manuscript. All authors contributed to manuscript revision, read and approved the submitted version.

\subsection{Acknowledgements}

Not applicable.

\section{References}

1. Bray F, Ferlay J, Soerjomataram I, Siegel RL, Torre LA, Jemal A: Global cancer statistics 2018: GLOBOCAN estimates of incidence and mortality worldwide for 36 cancers in 185 countries. CA: A Cancer Journal for Clinicians 2018, 68(6):394-424.

2. Miller KD, Nogueira L, Mariotto AB, Rowland JH, Yabroff KR, Alfano CM, Jemal A, Kramer JL, Siegel RL: Cancer treatment and survivorship statistics, 2019. CA: A Cancer Journal for Clinicians 2019, 69(5):363-385.

3. Dekker E, Tanis PJ, Vleugels JLA, Kasi PM, Wallace MB: Colorectal cancer. The Lancet 2019, 394(10207):1467-1480.

4. Van Der Stok EP, Spaander MCW, Grünhagen DJ, Verhoef C, Kuipers EJ: Surveillance after curative treatment for colorectal cancer. NAT REV CLIN ONCOL 2017, 14(5):297-315.

5. Brenner H, Kloor M, Pox CP: Colorectal cancer. The Lancet 2014, 383(9927):1490-1502.

6. Rosich L, Colomer D, Roué G: Autophagy controls everolimus (RAD001) activity in mantle cell lymphoma. AUTOPHAGY 2013, 9(1):115-117.

7. Kongara S, Karantza V: The interplay between autophagy and ROS in tumorigenesis.FRONT ONCOL 2012, 2.

8. Carroll RG, Martin SJ: Autophagy in multiple myeloma: What makes you stronger can also kill you. CANCER CELL 2013, 23(4):425426.

9. Subramanian A, Tamayo P, Mootha VK, Mukherjee S, Ebert BL, Gillette MA, Paulovich A, Pomeroy SL, Golub TR, Lander ES et al: Gene set enrichment analysis: A knowledge-based approach for interpreting genome-wide expression profiles. P NATL ACAD SCI USA 2005, 102(43):15545-15550.

10. Liberzon A, Subramanian A, Pinchback R, Thorvaldsdottir H, Tamayo P, Mesirov JP: Molecular signatures database (MSigDB) 3.0. BIOINFORMATICS 2011, 27(12):1739-1740.

11. Liberzon A, Birger C, Thorvaldsdóttir H, Ghandi M, Mesirov J, Tamayo P: The Molecular Signatures Database Hallmark Gene Set Collection. CELL SYST 2015, 1(6):417-425.

12. Newman AM, Liu CL, Green MR, Gentles AJ, Feng W, Xu Y, Hoang CD, Diehn M, Alizadeh AA: Robust enumeration of cell subsets from tissue expression profiles. NAT METHODS 2015, 12(5):453-457.

13. Markle B, May EJ, Majumdar APN: Do nutraceutics play a role in the prevention and treatment of colorectal cancer? CANCER METAST REV2010, 29(3):395-404.

14. Mokarram P, Albokashy M, Zarghooni M, Moosavi MA, Sepehri Z, Chen QM, Hudecki A, Sargazi A, Alizadeh J, Moghadam AR et al: New frontiers in the treatment of colorectal cancer: Autophagy and the unfolded protein response as promising targets. AUTOPHAGY 2017, 13(5):781-819.

15. Koustas E, Sarantis P, Kyriakopoulou G, Papavassiliou AG, Karamouzis MV: The interplay of autophagy and tumor microenvironment in colorectal cancer-ways of enhancing immunotherapy action. CANCERS 2019, 11(4). 
16. Yang HD, Kim HS, Kim SY, Na MJ, Yang G, Eun JW, Wang HJ, Cheong JY, Park WS, Nam SW: HDAC6 Suppresses Let-7i-5p to Elicit TSP1/CD47-Mediated Anti-Tumorigenesis and Phagocytosis of Hepatocellular Carcinoma. HEPATOLOGY 2019, 70(4):1262-1279.

17. Chen M, Lin Y, Liao Y, Liou J, Chen C: MPT0G612, a Novel HDAC6 Inhibitor, Induces Apoptosis and Suppresses IFN-gamma-Induced Programmed Death-Ligand 1 in Human Colorectal Carcinoma Cells. CANCERS 2019, 11(161710).

18. Hu F, Sun X, Li G, Wu Q, Chen Y, Yang X, Luo X, Hu J, Wang G: Inhibition of SIRT2 limits tumour angiogenesis via inactivation of the STAT3/VEGFA signalling pathway. Cell Death and Disease 2019, 10(1).

19. Zhang LL, Zhan L, Jin YD, Min ZL, Wei C, Wang Q, Chen YJ, Wu QM, Hu XM, Yuan Q: SIRT2 mediated antitumor effects of shikonin on metastatic colorectal cancer. EUR J PHARMACOL 2017, 797:1-8.

20. Zhao Y, Yang J, Liao W, Liu X, Zhang H, Wang S, Wang D, Feng J, Yu L, Zhu WG: Cytosolic FoxO1 is essential for the induction of autophagy and tumour suppressor activity. NAT CELL BIOL 2010, 12(7):665-675.

21. Nichols $\mathrm{PH}$, Ward U, Ramsden CW, Primrose JN: The effect of 5-fluorouracil and alpha interferon and 5-fluorouracil and leucovorin on cellular anti-tumour immune responses in patients with advanced colorectal cancer. BRIT J CANCER 1994, 70(5):946-949.

22. Eguchi J, Hiroishi K, Ishii S, Mitamura K: Interferon-alpha and interleukin-12 gene therapy of cancer: interferon-alpha induces tumorspecific immune responses while interleukin-12 stimulates non-specific killing. CANCER IMMUNOL IMMUN2003, 52(6):378-386.

23. De Simone V, Franzè E, Ronchetti G, Colantoni A, Fantini MC, Di Fusco D, Sica GS, Sileri P, MacDonald TT, Pallone F et al: Th17-type cytokines, IL-6 and TNF-a synergistically activate STAT3 and NF-kB to promote colorectal cancer cell growth. ONCOGENE 2015, 34(27):3493-3503.

24. Park JH, Van Wyk H, McMillan DC, Quinn J, Clark J, Roxburgh CSD, Horgan PG, Edwards J: Signal transduction and activator of transcription-3 (STAT3) in patients with colorectal cancer: Associations with the phenotypic features of the tumor and host. CLIN CANCER RES 2017, 23(7):1698-1709.

25. Giordano G, Parcesepe P, D'Andrea MR, Coppola L, Di Raimo T, Remo A, Manfrin E, Fiorini C, Scarpa A, Amoreo CA et al: JAK/Stat5mediated subtype-specific lymphocyte antigen 6 complex, locus G6D (LY6G6D) expression drives mismatch repair proficient colorectal cancer. Journal of Experimental and Clinical Cancer Research 2019, 38(1).

26. Wing K, Sakaguchi S: Regulatory T cells exert checks and balances on self tolerance and autoimmunity. NAT IMMUNOL 2010, 11(1):7-13.

27. Edin S, Wikberg ML, Dahlin AM, Rutegård J, Öberg Å, Oldenborg PA, Palmqvist R: The Distribution of Macrophages with a M1 or M2 Phenotype in Relation to Prognosis and the Molecular Characteristics of Colorectal Cancer. PLOS ONE 2012, 7(10).

28. Shapouri-Moghaddam A, Mohammadian S, Vazini H, Taghadosi M, Esmaeili SA, Mardani F, Seifi B, Mohammadi A, Afshari JT, Sahebkar A: Macrophage plasticity, polarization, and function in health and disease. J CELL PHYSIOL 2018, 233(9):6425-6440.

29. Chen P, Cescon M, Bonaldo P: Autophagy-mediated regulation of macrophages and its applications for cancer. AUTOPHAGY 2014, 10(2):192-200.

\section{Figures}




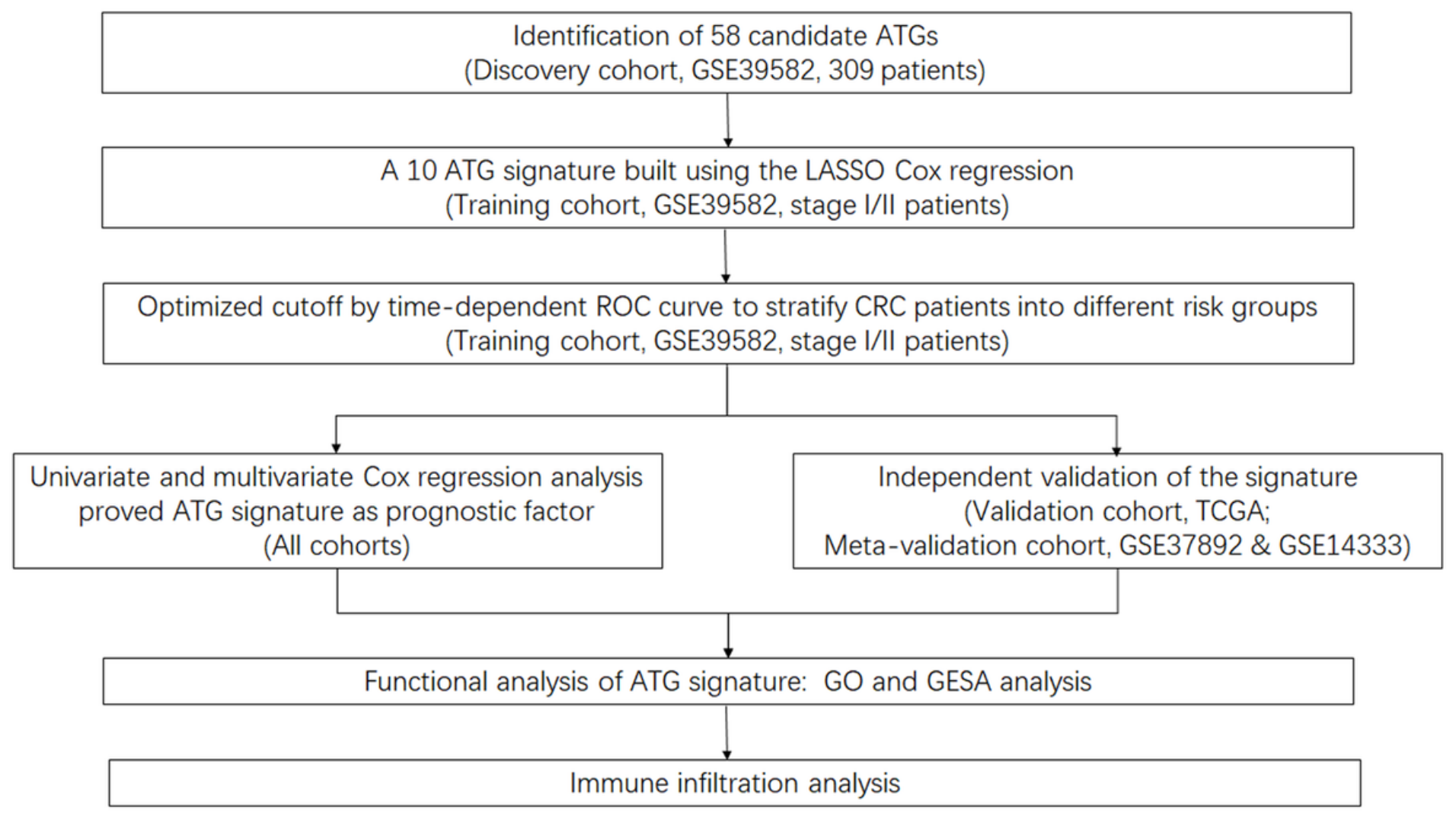

Figure 1

Flow chart

(A)

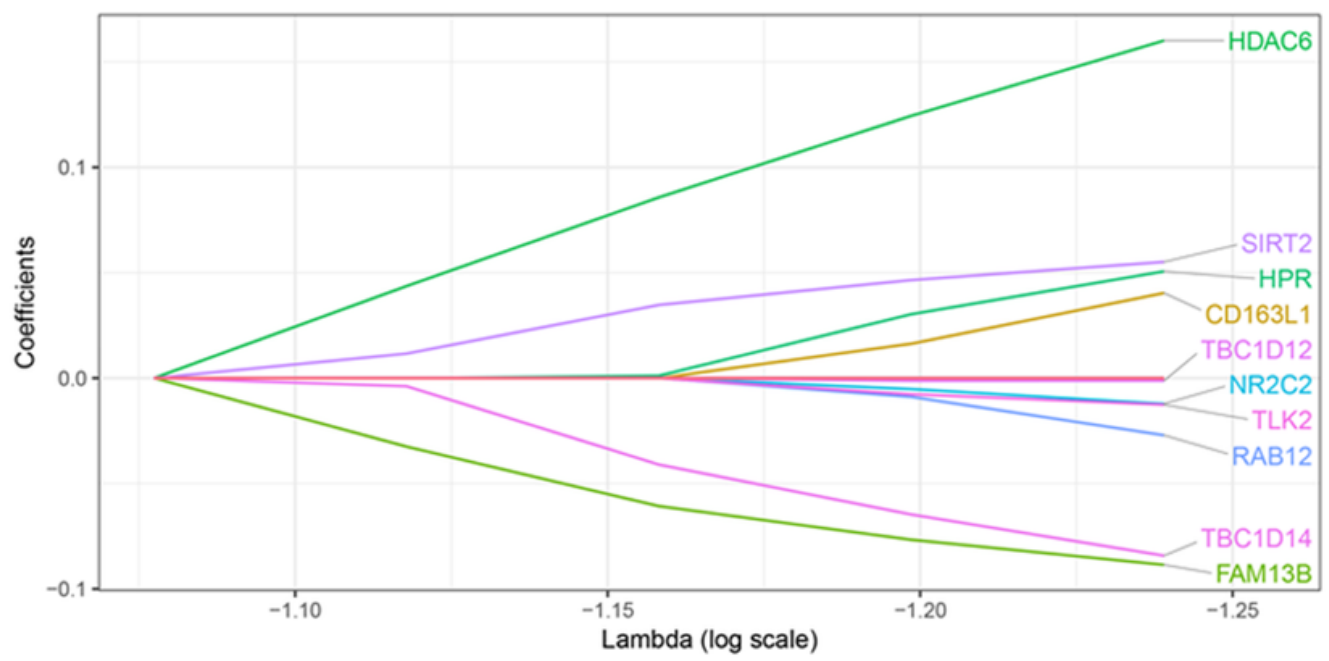

(B)

CIT/GSE39582

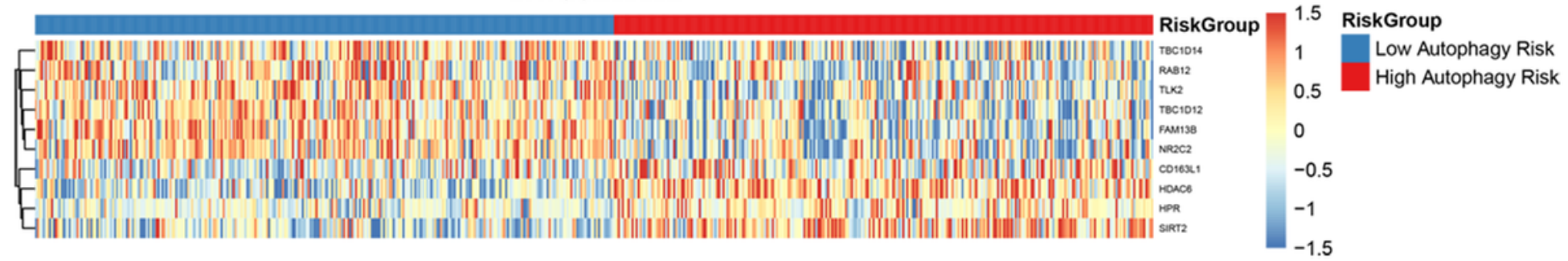


Figure 2

Discovered ATG signature for prognostic prediction of colorectal cancer. (A). Ten ATGs signature found from the LASSO Cox regression.

(B). Heatmap of the identified 10-gene signature.

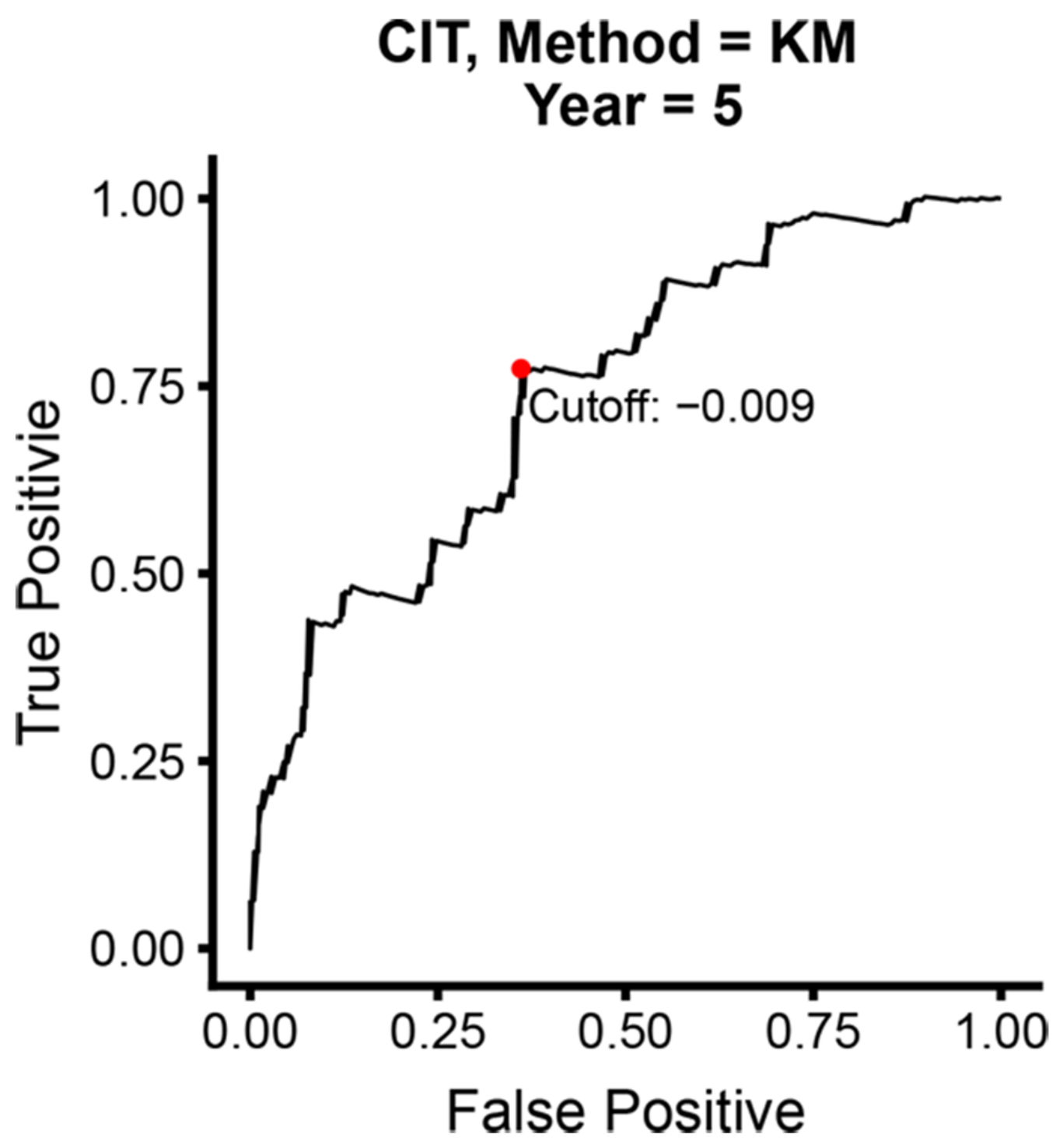

Figure 3

Time-dependent ROC curve for 5-year disease-free survival in stage I/II CRC patients in training cohort. 

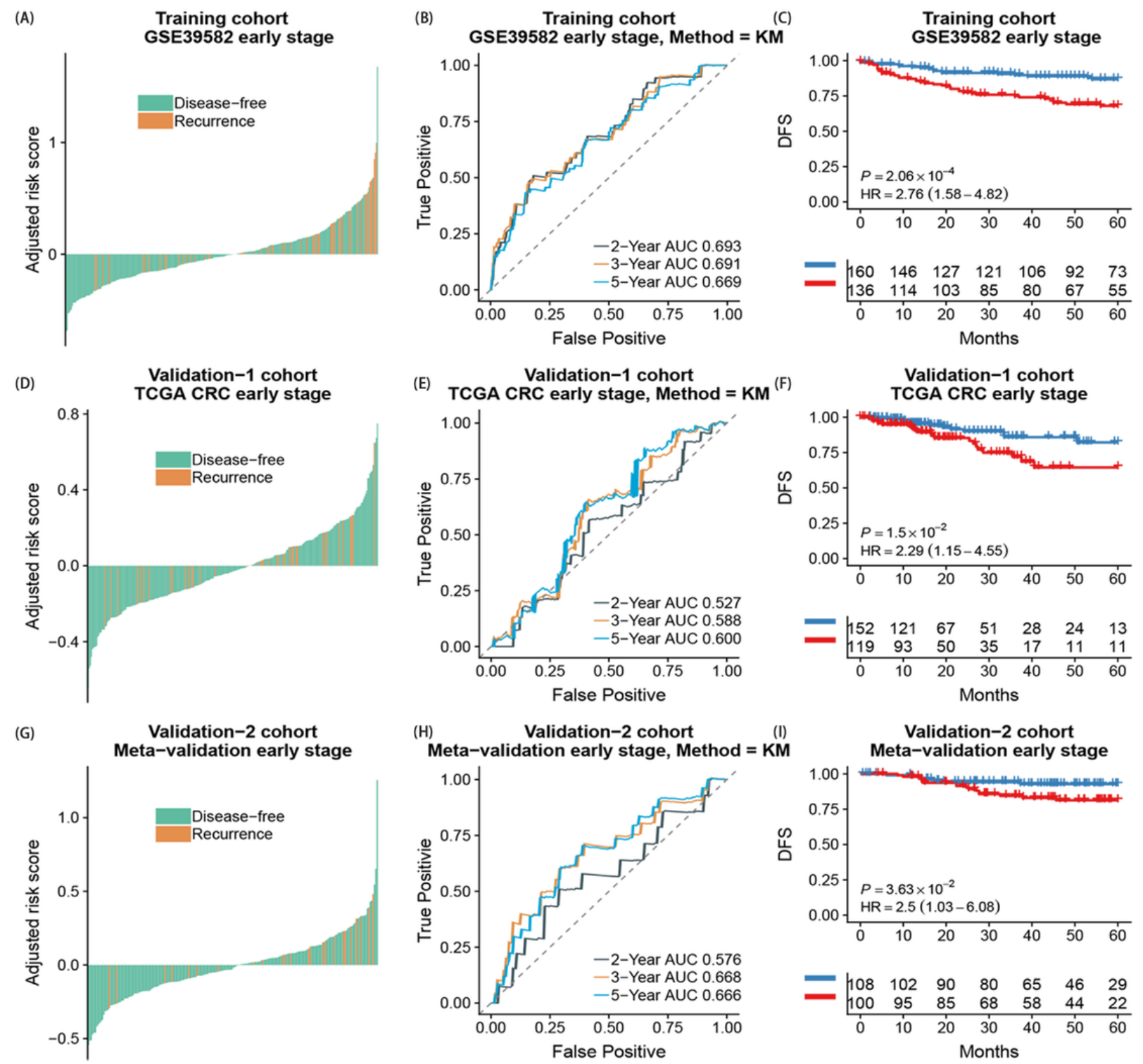

\section{Figure 4}

The association of the ATG signature with DFS in early stage patients with CRC. Patients with CRC of early stage were ranked by autophagy risk scores in the training cohort (A), the TCGA cohort (D) and the meta-validation cohort (G). Time-dependent ROC curves for DFS in early stage (stage I\&II) patients achieved with different duration in the training cohort (B), the TCGA cohort (E) and the metavalidation cohort $(\mathrm{H})$. Kaplan-Meier curves showed DFS of early stage patients in low and high autophagy groups in training cohort(C), the TCGA cohort (F) and meta-validation cohort (I), respectively. P values comparing risk groups were calculated with the log-rank test. Hazard ratios (HRs) and $95 \%$ Cls are for low vs high autophagy risk. CRC = colorectal cancer, ATG = autophagy-related gene, DFS = disease-free survival. 
(A)

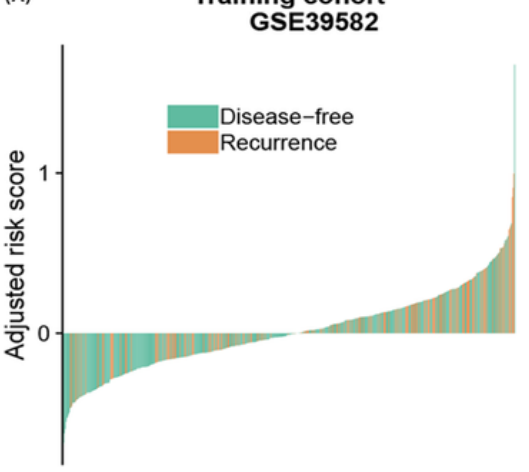

(D)
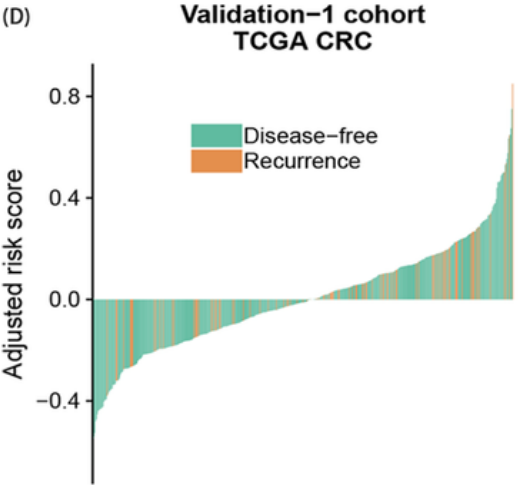

(G)

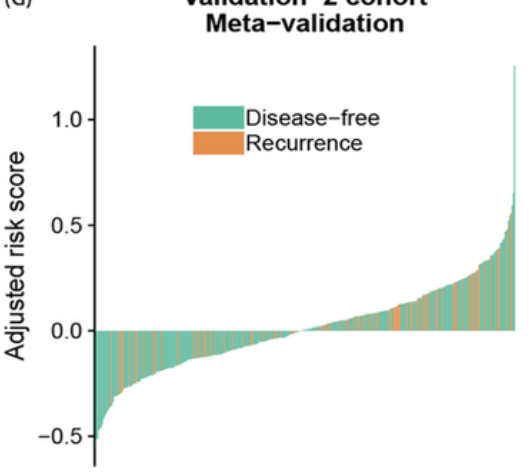

(B)

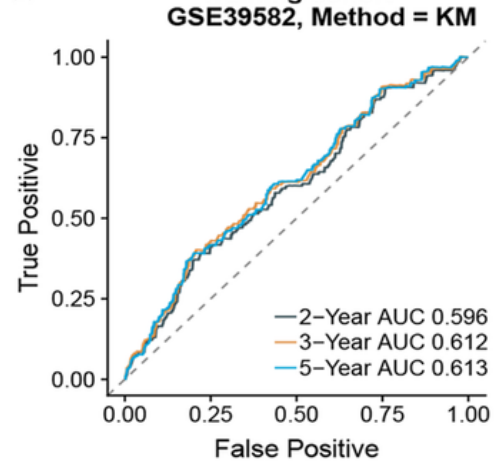

(E)

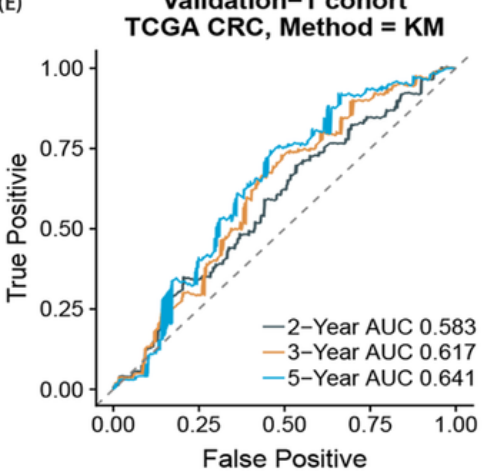

(H)

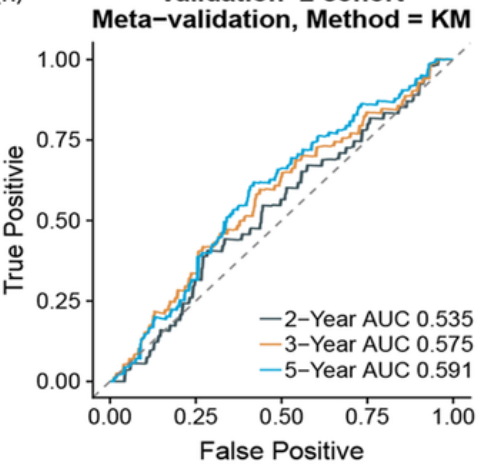

(C)

Training cohort GSE39582

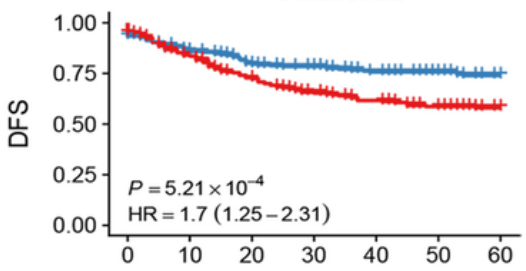

\begin{tabular}{ccccccc}
289 & 236 & 199 & 184 & 163 & 140 & 112 \\
268 & 215 & 178 & 148 & 130 & 109 & 90 \\
\hline 0 & 10 & 20 & 30 & 40 & 50 & 60 \\
\hline & \multicolumn{5}{c}{$\begin{array}{c}1 \\
\text { Months }\end{array}$} &
\end{tabular}

(F)

Validation-1 cohort TCGA CRC

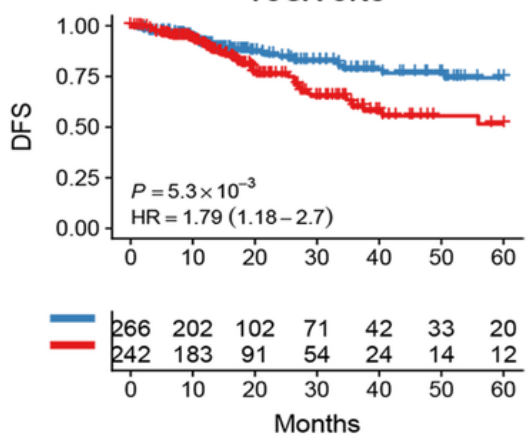

(I) Validation-2 cohort Meta-validation

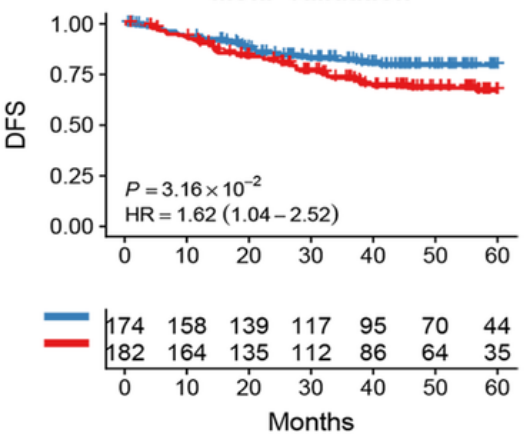

\section{Figure 5}

The association of the ATG signature with DFS in CRC patients with all stages. Patients with CRC of early stage were ranked by autophagy risk scores in the training cohort (A), the TCGA cohort (D) and the meta-validation cohort (G). Time-dependent ROC curves for DFS in CRC patients achieved with different duration in the training cohort (B), the TCGA cohort (E) and the meta-validation cohort (H). Kaplan-Meier curves showed DFS of CRC patients in low and high autophagy groups in training cohort (C), the TCGA cohort (F) and meta-validation cohort (I), respectively. P values comparing risk groups were calculated with the log-rank test. Hazard ratios (HRs) and $95 \%$ Cls are for low vs high autophagy risk. $\mathrm{CRC}=$ colorectal cancer, $\mathrm{ATG}=$ autophagy-related gene, DFS = disease-free survival. 
(A)

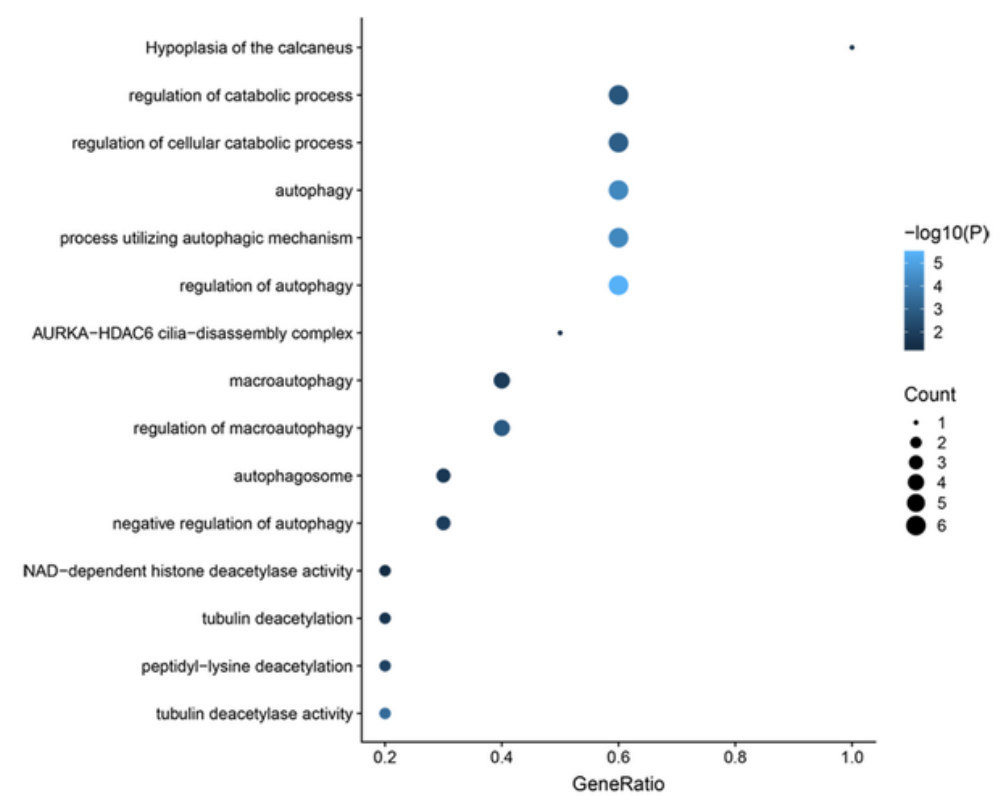

(B)
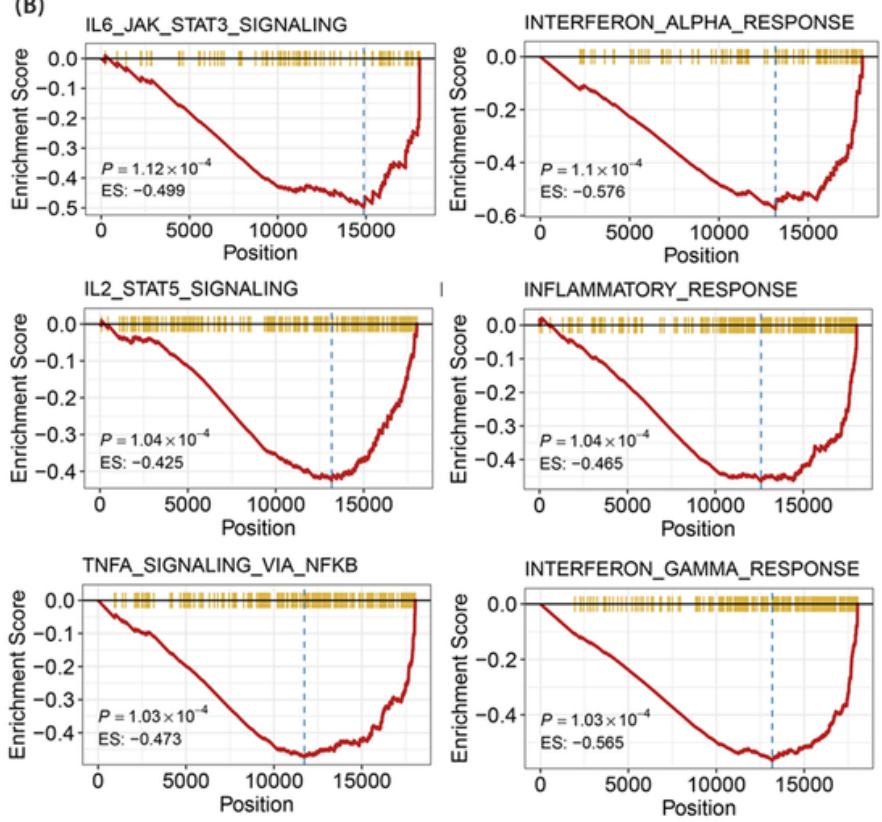

Figure 6

Functional analysis of the 10 autophagy signature genes. (A). Gene ontology (GO) of the 10 autophagy signature genes. (B). GSEA showed some immune/ inflammatory pathways downregulated in high autophagy risk patients.

(A)

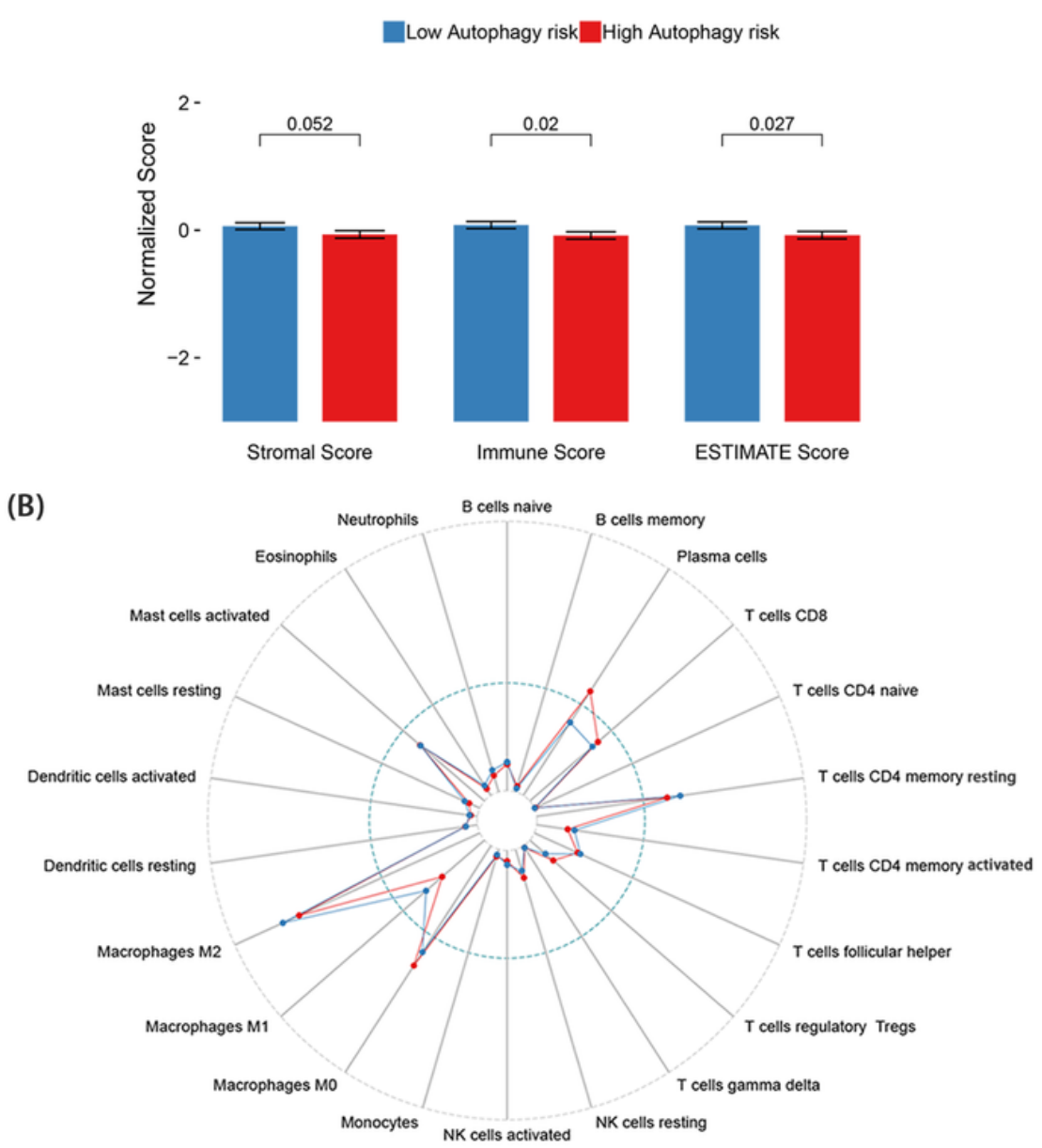

(C)
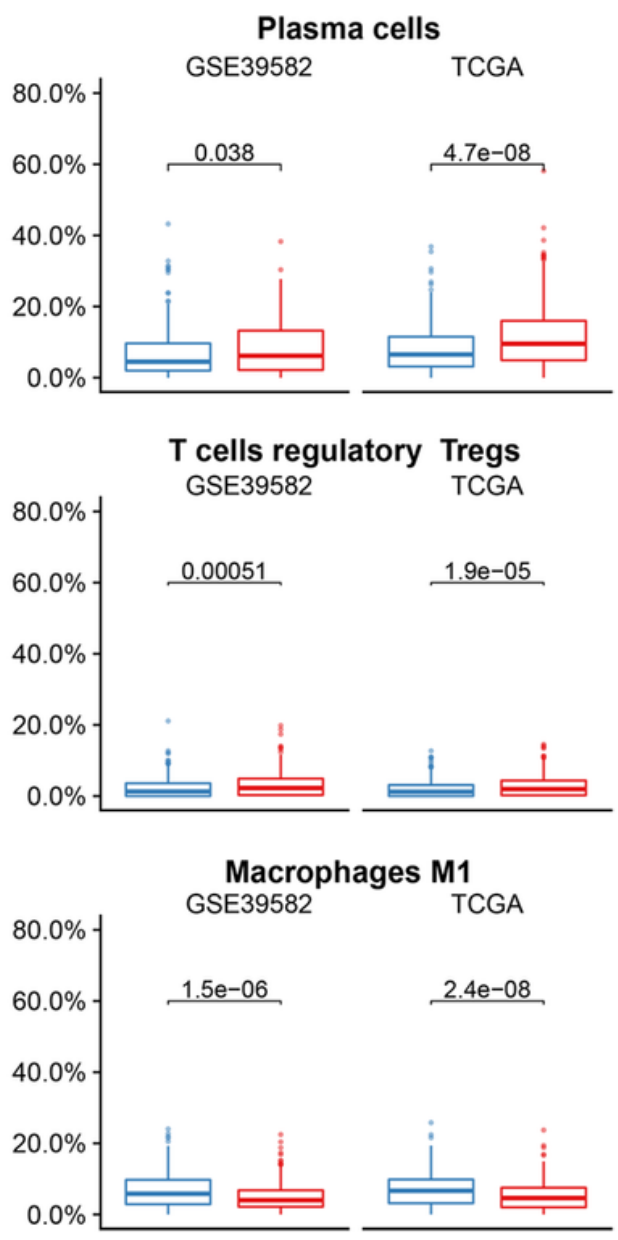
Immune infiltration status of ATG signature risk groups. (A). ESTIMATE algorithm in the TCGA cohort. (B). 22 immune cells' abundance for different immune risk groups. (C). Plasma cells and T cells regulatory were enriched in the high-risk group. The macrophage M1 was enriched in the low-risk group. P-values are based on the Wilcoxon Test.

A

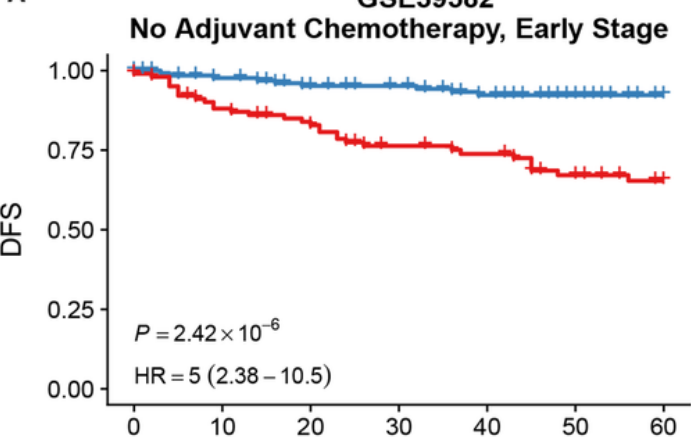

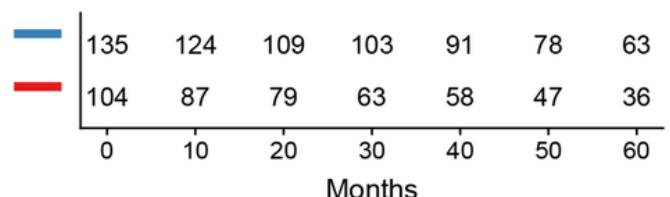

C

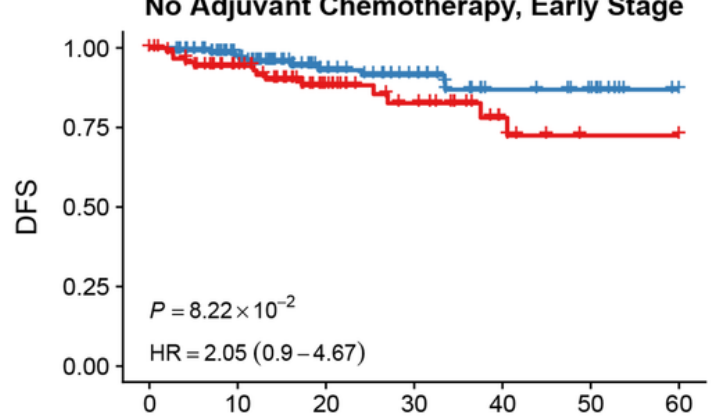

B $\quad$ GSE39582

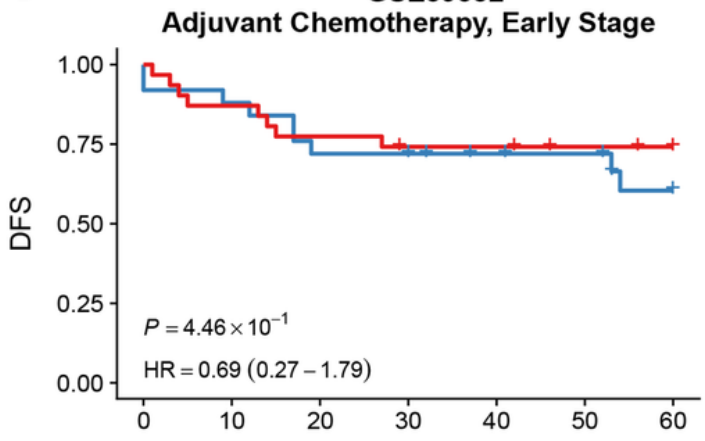

$-$\begin{tabular}{rrrrrrr}
25 & 22 & 18 & 18 & 15 & 14 & 10 \\
31 & 27 & 24 & 22 & 22 & 20 & 19 \\
\hline 0 & 10 & 20 & $\begin{array}{c}30 \\
\text { Months }\end{array}$ & 40 & 50 & 60
\end{tabular}

D

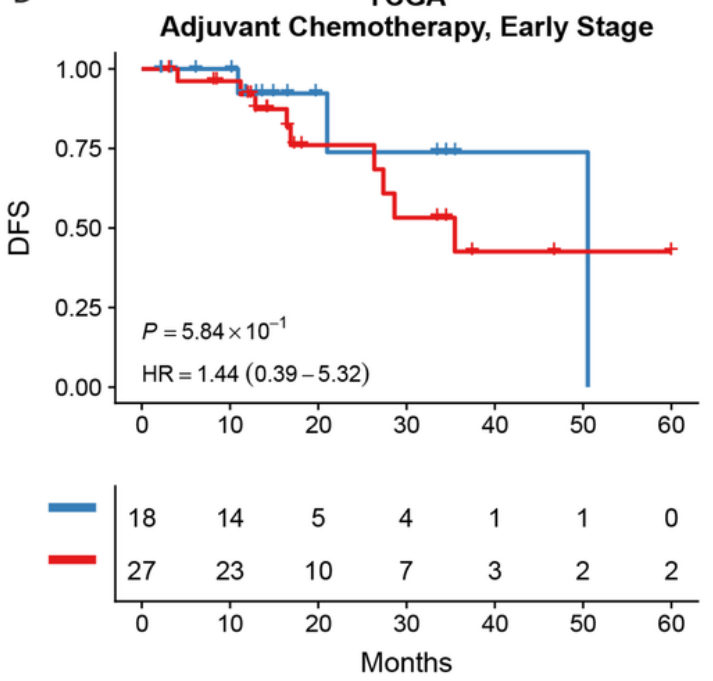

\section{Figure 8}

Effects of adjuvant chemotherapy between different autophagy risk groups in early stage CRC patients. Kaplan-Meier curves showed DFS of early stage patients between low and high autophagy groups with and without adjuvant chemotherapy respectively in the GSE39582(A and B) cohort and the TCGA cohort(C and D). P values comparing risk groups were calculated with the log-rank test. Hazard ratios (HRs) and $95 \%$ Cls are for low vs high autophagy risk. CRC = colorectal cancer, DFS $=$ disease-free survival.

\section{Supplementary Files}

This is a list of supplementary files associated with this preprint. Click to download.

- SupplementaryTable2.xlsx

- SupplementaryTable1.xlsx 